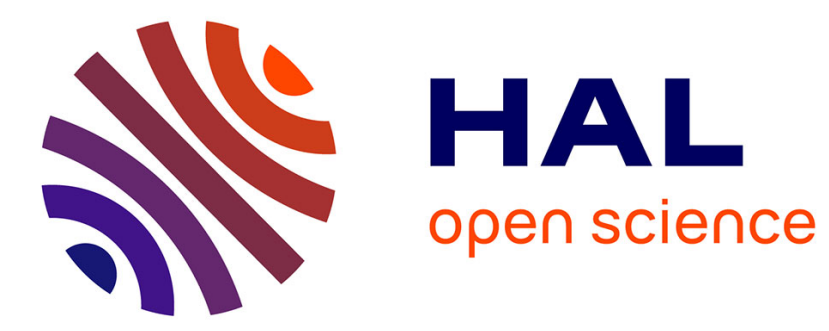

\title{
Model Order Reduction for Problems with large Convection Effects
}

Nicolas Cagniart, Yvon Maday, Benjamin Stamm

\section{To cite this version:}

Nicolas Cagniart, Yvon Maday, Benjamin Stamm. Model Order Reduction for Problems with large

Convection Effects. 2016. hal-01395571

\section{HAL Id: hal-01395571 \\ https://hal.sorbonne-universite.fr/hal-01395571}

Preprint submitted on 10 Nov 2016

HAL is a multi-disciplinary open access archive for the deposit and dissemination of scientific research documents, whether they are published or not. The documents may come from teaching and research institutions in France or abroad, or from public or private research centers.
L'archive ouverte pluridisciplinaire HAL, est destinée au dépôt et à la diffusion de documents scientifiques de niveau recherche, publiés ou non, émanant des établissements d'enseignement et de recherche français ou étrangers, des laboratoires publics ou privés. 


\title{
Model Order Reduction for Problems with large Convection Effects
}

\author{
Nicolas Cagniart, Yvon Maday and Benjamin Stamm
}

\begin{abstract}
The reduced basis method allows to propose accurate approximations for many parameter dependent partial differential equations, almost in real time, at least if the Kolmogorov $n$-width of the set of all solutions is small, under variation of the parameters. The idea is that any solutions may be well approximated by the linear combination of some well chosen solutions that are computed once and for all (by another, more expensive, discretization) for some well chosen parameter values. In some cases however, such as for problems with large convection effects, the linear representation is not sufficient and, as a consequence, the set of solutions needs to be transformed/twisted so that the combination of the proper twist and the appropriate linear combination recovers an accurate approximation. This paper presents a simple approach towards this direction, preliminary simulations support this approach.
\end{abstract}

\section{Introduction}

Fast reliable solutions to many queries parametric Partial Differential Equations (PDE) have many applications among which real time systems, optimization problems and optimal control. Many different methods for reducing the complexity of

Nicolas Cagniart

Sorbonne Universits, UPMC Univ Paris 06, UMR 7598, Laboratoire Jacques-Louis Lions, F75005, Paris, France, e-mail: cagniartn@1jll.math.upmc.fr

Yvon Maday

Sorbonne Universits, UPMC Univ Paris 06, UMR 7598, Laboratoire Jacques-Louis Lions, F75005, Paris, France \& Institut Universitaire de France \& Division of Applied Mathematics, Brown University. e-mail: maday@ann.jussieu.fr

Benjamin Stamm

Center for Computational Engineering Science, RWTH Aachen University, Aachen, Germany and Computational Biomedicine, Institute for Advanced Simulation IAS-5 and Institute of Neuroscience and Medicine INM-9, Forschungszentrum Jülich, Germany. e-mail: stamm@mathcces.rwth-aachen.de. 
the computations when such many queries are required have blossomed for answering this specific need. One of the approaches that have emerged is reduced order modeling (ROM). Methods in this category have been developed and are now well understood and set on firm grounds.

The reduced basis method, which is the method that we focus on in this paper, enters in this frame and consists in, i) defining a sequence of low dimensional spaces for the approximation of the whole set of all solutions to the parametric PDE when the parameters vary (called hereafter the solution manifold associated to our problem); ii) once such a sequence of low dimensional spaces (known as reduced basis spaces) is known, an approximate solution is sought in such a chosen reduced space to the PDE for the values of the parameter we are interested in. The approximation is often based on a Galerkin formulation. For such reduced basis methods, both the variety of applications and the theory are now quite sound. For instance, reliable algorithms with a priori estimates and certified a posteriori errors have been developed for elliptic and parabolic problems, with or without so-called affine parameter dependence, see e.g. the two recent books on the subject [12] and [20] and, of course, the publications therein.

Reduced basis methods, classically, consider the solution manifold associated to the parametrized problem as outlined above and are appropriate if this manifold can be approximated accurately by a sequence of finite dimensional spaces. The mathematical frame for this is inherently linked to the notion of Kolmogorov width of solution manifolds, i.e. on how well the solution manifold can be approached by a finite dimensional linear space. More precisely, let $\mathscr{M}$ be a manifold embedded in some normed linear space $X$. The Kolmogorov n-width of $\mathscr{M}$ is defined as:

$$
d_{n}(\mathscr{M}, X)=\inf _{E_{n}} \sup _{f \in \mathscr{M}^{g \in E_{n}}}\|f-g\|_{X}
$$

The first infimum being taken over all linear subspaces $E_{n}$ of dimension $n$ embedded in $X$.

Even if, from the practical point of view, there are various ways of checking that this is the case, the first natural mathematical question is to provide an estimation of the Kolmogorov n-width of $\mathscr{M}$. Second, the question of an applied mathematician is if one can actually build an optimal, or close to optimal sequence of basis sets for these spaces?

Of course, in the vast majority of real cases, there is no analytical expression for this dimension but there are some papers giving bounds for some restricted classes of problems in the literature. For instance, in [17] bounds on $d_{n}$ are found for solution manifolds corresponding to regular elliptic problems and where the parameter dependence is on the forcing term. More general cases can be handled using the results in [8]. The hypothesis therein is on the regularity of the solution with respect to the parameter dependence, it is proven that, under analyticity assumption on the behavior of the parameters in the PDE, the small Kolmogorov n-width of the manifold of parameters $\mathscr{C}\left(\leq c n^{-t}, t>1\right)$ implies the smallness of the Kolmogorov n-width of the associated solutions manifold $\mathscr{M}_{\mathscr{C}}\left(\leq c n^{-s}, s \leq t-1\right)$. 
In practice, instead of the "optimal" linear subspace of dimension $n$ in the sense described earlier, we build a "good" linear subspace. In the literature, the two most classical algorithms are the greedy method based on a certified (or at least fair enough) a posteriori estimator, and the Proper Orthogonal Decomposition (POD). We proceed assuming that the chosen algorithm has given a "good" basis "close" to the optimal one, that is, we assume that our reduced family of spaces $\left\{X_{n}\right\}_{n}$ satisfies:

$$
d_{n}(\mathscr{M}, X) \approx \sup _{f \in \mathscr{M} \in X_{n}}\|f-g\|_{X}
$$

A first paper on this subject is [16], where the authors derived error bounds on the error for the Reduced Basis Method (RBM) approximation in case of a single parameter dependent elliptic PDE. More general results have been obtained more recently for the greedy approach of the RBM $[3,10]$. The optimality considered in the case of POD is slightly different. The POD focuses on minimizing the average error (parameter wise), in some norm. More precisely, we have the well known relation

$$
\int_{\mathscr{C}}\left\|u(\mu)-\Pi_{P O D} u(\mu)\right\|^{2} d \mu=\sum_{i>N_{P O D}} \lambda_{i}
$$

where $\Pi_{P O D}$ is the orthogonal projection onto the POD reduced space of dimension $N_{P O D}$ and the $\lambda_{i}$ are the eigenvalues of the associated correlation operator, in decreasing order. The faster the decay of the eigenvalues, the fewer modes are needed for a good (in average) reconstruction of the solution manifold.

Up to now, most of the literature on the subject, deals with problems where one can expect/check/prove/ or hope, that the solution manifold $\mathscr{M}_{\mathscr{C}}$ has a small Kolmogorov $n$-width. There are however cases where the plain approach does not work and some transformation of $\mathscr{M}_{\mathscr{C}}$ needs to be done. An example is for instance the use of the Piola transform in the processing of the velocity field when the PDE is the Stokes or Navier Stokes problem and the parameter includes the geometry of the computational problem (see e.g. [14]).

The most classical and simple example illustrating limitations of reduced models due to large Kolmogorov n-width is the pure transport equation, with constant speed $c>0$. Formally, we consider the following parametric PDE over the domain $\Omega=$ $(a, b) \subset \mathbb{R}$

$$
\left\{\begin{array}{lll}
\partial_{t} u(x, t)+c \partial_{x} u(x, t) & =0, & \text { in } \Omega \times] 0, T[ \\
u(x, 0) & =u_{0}(x), & \text { in } \Omega \\
c \in \mathscr{C}:=\left[c_{\text {min }}, c_{\text {max }}\right] . &
\end{array}\right.
$$

The analytic solution is given by

$$
u(x, t ; c)=u_{0}(x-c t) .
$$

We can consider two solution manifolds. Either the space time solution manifold

$$
\mathscr{M}_{\mathscr{C}}^{x, t}=\{u(\cdot, \cdot ; c), c \in \mathscr{C}\},
$$

or a more natural solution manifold in our context is the snapshot solution manifold 


$$
\mathscr{M}_{\mathscr{C}}^{x}=\{u(\cdot, t ; c), t \in[0, T], c \in \mathscr{C}\} .
$$

We will first give an illustrative idea of $d_{n}\left(\mathscr{M}_{c}^{x}\right)$, i.e for a fixed convection parameter. The only "parameter" left thus time and $d_{n}\left(\mathscr{M}_{c}^{x}\right)$ is, of course, smaller than $d_{n}\left(\mathscr{M}_{\mathscr{C}}^{x}\right)$.

Suppose now that our initial solution is compactly supported and let $\ell$ denote the Lebesgue measure of its support. Let us assume in addition that its support is included in $] a, a+\ell\left[\right.$. Then, there are at least $(b-a) / \ell$ snapshots $\left\{u\left(\cdot, t^{k} ; c\right)\right\}_{k}$ obtained for $t_{k}=k \ell / c$ that are two by two orthogonal proving that a lower bound of the Kolmogorov n-width is $(b-a) / \ell$. For a given accuracy, reducing $\ell$, we can make the size of the reduced basis needed arbitrarily large. Another example of badly behaved manifold space can also be found in [22].

Our objective is to use the fact that, apart from a translation, the solution manifold for the whole time simulation can be represented by a unique basis. However, let us stress that this translation is not a linear process hence the Kolmogorov process cannot capture it. An additional ingredient to existing reduced order methods has thus to be added so as to capture this very simple problem structure.

Most of the works in the reduced order modeling community on convection dominated problem have been done on the stabilization issue, and not on the reduction of the Kolmogorov n-wdith. For instance, the authors in [9] have proven that using, as usual, the residual of the PDE as a surrogate for the true error, is not adapted if convection is dominating as the relative a posteriori estimator is not fair enough. Their method involves other norms than the natural ones, and increases the stability at each iteration by enriching the trial space. Once again, their method improves the stability of the construction of a reduced basis, but does not handle the fact that the solution manifold can have a large Kolmogorov n-width.

In the same direction let us quote the papers related to the so called GNAT approach [6], [7] where the authors propose also an alternative reduction approach for these type of problems.

In [1], the authors address the stability issue in another direction. They give ideas and show numerical examples illustrating the fact that using $L^{1}$-minimisation, instead of the - more classical $-L^{2}$-minimisation (corresponding to a Galerkin scheme, which is natural in the reduced modeling context), does a better job for handling shocks (as appears in non linear convection problems) and provides more stable results. However this approach does not cure the problem that we have indicated above related to the large dimension of the solution manifold.

Let us also mention at this level, as an intermediate approach, the paper [5]. As standard reduced order modeling fails, the author chooses, in a preprocessing step, to "chop off" the reduced basis functions resulting in a kind of adaptive coarse enriched finite element method.

Very few papers takle the n-width issue directly. In [22], the authors propose a method that is the first attempt to use shock fitting related ideas in the context of reduced order modeling. The idea is to decompose the spatial domain into zones separated by shocks. In each zone, classical reduced order modeling is performed, and the shocks dynamic is handled using another equation. For them, it is given by 
Rankine-Hugoniot conditions. This method, just as any other shock fitting method, is somehow limited to one dimensional problems.

In [11], the authors develop a method where the POD basis is reconstructed at each time step to follow the propagation of the phenomenon. More precisely, by referring to Lax-pairs, they choose as reduced basis the modes of the Schrödinger operator where the potential is taken as the solution at the previous time step. Even if no theoretical proof of this ansatz is presented, the numerical results presented in that paper illustrate the interest of the approach for selecting the reduced space and adding stability to the process without curing however the large increase of the dimension of the reduced space when the accuracy requirement increases.

The method presented in [13] is similar to our work in many aspects, in particular in looking for a change of variable for better representing the solution manifold. Their approach relies on the existence of a main mode $u_{0}$ that, by convection, represents most of the solution. The proper change of variable (written as a sum of advection modes) is fitted by evaluating Wasserstein distances between the snapshots in $\mathscr{M}_{\mathscr{C}}^{x}$, with modes being obtained by solving Monge-Kantorovich optimal transport problems w.r.t. the reference mode $u_{0}$. Various numerical results illustrate the approach, however only in cases where the solution exhibits indeed such a main mode $u_{0}$ which is doubtful in nonlinear processes. We will come back on their ideas in the following sections.

The last approach in this direction developed in [2] and in [19] uses the same initial idea. Their formal and general presentation is quite interesting and enlightening, however the restrictions imposed on the formulation of the transformed equations seems to be somhow too stringent for many reduction processes.

This paper is the first of a series where we develop our approach in different situations. We start here by presenting the general framework and notations. We introduce the notion of "preconditioning" of a solution manifold based on our knowledge of the process (differing here somehow from the optimal transport problem approach in [13]). We then apply our method to the specific problem of the one dimensional unsteady viscous Burger equation and we then present some numerical simulations confirming the feasibility of the method. The end of the paper states some perspectives.

\section{Formal presentation}

Let us consider a general time dependent parametric PDE in some physical space $\Omega \subset \mathbb{R}^{d}, d=1,2,3$

$$
\left\{\begin{array}{lrl}
u_{t}+\mathscr{L}(u ; \mu) & =0 & \text { in }[0, T] \times \Omega \\
u(\cdot, t=0 ; \mu) & =u_{0}(\cdot, \mu) & \text { in } \Omega \\
B(u ; \mu) & =0 & \text { on } \partial \Omega
\end{array}\right.
$$


where $\mu$ varies in some compact parameter space $\mathscr{C}$. Our approach considers the corresponding snapshot solution manifold $\mathscr{M}_{\mathscr{C}}^{x}$ as defined in (7) that is embedded in $X$, that, for the sake of conveniency, we choose equal to $L^{2}(\Omega)$.

Let us assume that the solution manifold has a simple structure, not reflected though by the Kolmogorov n-width but hidden by a transformation of the solution manifold. As stated in the introduction, we can think of the transport equation as being the simplest example for which this is occurring. The objective is to find, through a "preconditioning" step, how to recover the simple structure of the solution manifold.

In this "preconditioning" step, we target a family of (smooth) invertible mappings

$$
\mathscr{F}_{\mathscr{C}}=\{F: \bar{\Omega} \mapsto \bar{\Omega}\}
$$

in which there exists well chosen applications

$$
\begin{aligned}
{[0, T] \times \mathscr{C} } & \rightarrow \mathscr{F}_{\mathscr{C}} \\
(t, \mu) & \mapsto F_{t ; \mu}
\end{aligned}
$$

such that the corresponding preconditioned solution manifold, defined as:

$$
\mathscr{M}_{\mathscr{F}, \mathscr{C}}^{x}:=\left\{u\left(F_{t ; \mu}^{-1}(\cdot), t ; \mu\right), \mu \in \mathscr{C}, t \in[0, T]\right\}
$$

has a smaller Kolmogorov n-width. The definition of the set $\mathscr{F}_{\mathscr{C}}$ is based on a priori expertise on the behavior of the solution. We aim to conceive and design it during a preprocessing step (generally called "offline" in the RBM community).

In what follows, we explain how we use this preconditioned solution manifold and how we pick the correct application $F_{t ; \mu}$, in a computationally efficient way.

\subsection{Algorithm}

For simplicity, let us assume that we are using an explicit Euler scheme for the time discretization. Extensions to implicit, higher order time discretization, or more involved conservative numerical scheme, is straightforward and will be reported in a future paper ${ }^{1}$. Our semi-discretized PDE then becomes

$$
\left\{\begin{array}{llr}
\frac{u^{n+1}-u^{n}}{d t}+\mathscr{L}\left(u^{n} ; \mu\right) & =0 & \text { in } \Omega \\
u(\cdot, t=0 ; \mu) & =u_{0}(\cdot, \mu) & \text { in } \Omega \\
B\left(u^{n} ; \mu\right) & =0 & \text { on } \partial \Omega
\end{array}\right.
$$

Here, as is classical, $d t$ denotes the time step, and $u^{n}$ an approximation for the solution to (8) at time $n d t$.

\footnotetext{
${ }^{1}$ Note that, of course, this choice of an explicit scheme involves a limitation on the time step due to a CFL condition that can be severe for an accurate finite element or finite difference scheme but reveals to be moderate in the reduced basis framework.
} 
Assume we have a basis $\left\{\phi_{i}\right\}$ such that $\operatorname{span}\left\{\phi_{i}\right\}$ approaches to a given accuracy the preconditioned solution manifold $\mathscr{M}_{\mathscr{F}, \mathscr{C}}^{x}$ defined in (11). Since $\mathscr{M}_{\mathscr{F}, \mathscr{C}}^{x}$ is assumed to be of small Kolmogorov n-width, we expect that we can find such a basis of moderate size. At each time step, we look for coordinates $\left(\alpha_{i}^{n+1}\right)_{i}$ on the reduced basis and an application $F_{n+1} \in \mathscr{F}_{\mathscr{C}}$ such that $u\left(\cdot, t^{n+1} ; \mu\right)$ is well approximated by:

$$
u^{n+1}:=\sum_{i=1}^{M} \alpha_{i}^{n+1} \phi_{i} \circ F_{n+1} .
$$

In order to expect the search for $F_{n+1}$ be computationally tractable, let us assume that our family $\mathscr{F}_{\mathscr{C}}$ can be parametrized by a few parameters: that is

$$
\forall F_{t ; \mu} \in \mathscr{F}_{\mathscr{C}}, \quad \exists\left(\gamma_{j}\right)_{j}, \text { such that } F_{t ; \mu}=F\left[\gamma_{1}(t ; \mu), \ldots, \gamma_{m}(t ; \mu)\right] .
$$

In the discrete setting, the search for $F_{n+1}$ then reduces to the search for $\left(\gamma_{j}^{n+1}\right)_{j}$, and we set $F_{n+1}=F\left[\gamma_{1}^{n+1}, \ldots, \gamma_{m}^{n+1}\right]$.

We are thus simultaneously looking for a proper appropriate reduced space (defined as the span of the $\left.\left(\phi_{i} \circ F_{n+1}\right)_{i}\right)$ and for coordinates on this reduced space. We have chosen to derive our solution from some minimization problem of the form:

$$
\left(\gamma_{j}^{n+1}, \alpha_{i}^{n+1}\right)=\underset{\left(\gamma_{j}, \alpha_{i}\right)}{\operatorname{argmin}}\left\|\sum_{i} \alpha_{i} \phi_{i} \circ F\left(\left[\gamma_{j}\right]_{j}\right)-u^{n}+d t \mathscr{L}\left(u^{n} ; \mu\right)\right\|
$$

for some appropriate norm $\|\cdot\|$ on $X$.

Remark 1. It is interesting to note that our approach, in this context, may be presented as a shock fitting method, and thus one may fear that it will suffer from the classical drawback of this class of approach, especially the difficulty to generalize to multidimensional framework. One reassuring element is that the position of the fitting $F_{n}$ is not defined through the Rankine-Hugoniot conditions but through the minimization process (15), and the evolution in time can be chosen to follow any appropriate conservative numerical scheme (this does not mean, however, that the extension to two dimensional problems does not lead to some difficulties! This is under investigation and will be presented in a future paper (see also [4])).

Several choices are possible for the sense in which we will minimize this quantity. One example will be given in the next section. We propose the following generic algorithm.

Initialize $\alpha_{i}$ and $\gamma_{j}$

$$
\left(\alpha_{i}^{n+1,0}, \gamma_{j}^{n+1,0}\right)=\left(\alpha_{i}^{i n i}, \gamma_{j}^{i n i}\right)
$$

$\alpha_{i}^{i n i}$ and $\gamma_{j}^{i n i}$ will depend on the previous timesteps, namely on $\left(\alpha_{i}^{k}\right)_{i}$ and $\left(\gamma_{j}^{k}\right)_{j}$ for $k \leq n$. 
Then, assuming that $\left(\alpha_{i}^{n+1, q}, \gamma_{j}^{n+1, q}\right)$ are known for some internal iteration $q \geq 0$, we proceed

Fit the $\alpha_{i}$ given $\gamma_{j}^{n+1, q}$

Find $\left(\alpha_{i}^{n+1, q+1}\right)_{i}$ that minimizes the following quantity (in some sense):

$$
\sum_{i} \alpha_{i}^{n+1, q+1} \phi_{i} \circ F\left(\left(\gamma_{j}^{n+1, q}\right)_{j}\right)-u^{n}+d t * \mathscr{L}\left(u^{n} ; \mu\right)
$$

Fit the $\gamma_{j}$ given $\left(\alpha_{i}^{n+1, q+1}\right)_{i}$

Find $\left(\gamma_{j}^{n+1, q+1}\right)_{j}$ that minimizes the following quantity (in some sense):

$$
\sum_{i} \alpha_{i}^{n+1, q+1} \phi_{i} \circ F\left(\left(\gamma_{j}^{n+1, q+1}\right)_{j}\right)-u^{n}+d t * \mathscr{L}\left(u^{n} ; \mu\right)
$$

until convergence (for which, say $q=q^{*}$ ). Then, we set

$$
\left(\alpha_{i}^{n+1}, \gamma_{j}^{n+1}\right)=\left(\alpha_{i}^{n+1, q^{*}+1}, \gamma_{j}^{n+1, q^{*}+1}\right) .
$$

\subsection{Discussion}

The two closest methods to ours are first the one developed in $[2,19]$, second the one presented in [13].

In the former method, there is also the search for a phase component: $F(t ; \mu)$ and a shape component: $v:=u(\cdot, t ; \mu) \circ F(t ; \mu)$ that they name the "calibrated solution". They present the approach in the frame of Lie group action and thus introduce the notion of equivariance with respect to the group action for the calibrated solution. Hence, instead of best fitting these two objects from the discrete equation (12) by solving the optimal problem (15), the idea is to find an equation satisfied by the calibrated solution, i.e an operator $\tilde{\mathscr{L}}$ such that $v$ is solution to the following equation

$$
v_{t}+\tilde{\mathscr{L}}(v ; \mu)=0 \text { in }[0, T] \times \Omega
$$

To close the system, they need to add a well chosen equation on $F(t ; \mu)$. Well chosen here means that the calibrated equation has to be well posed, and the dynamics of the shape component should be much simpler than those of the original solution.

The second paper [13] differs on two points. There is a unique reference mode $u_{0}$ that allows to characterize the mapping, obtained from an optimal transport problem. The interest is that there is no need to have an expertise on what is the set $\mathscr{F}_{\mathscr{C}}-$ 
the Monge-Kantorovich optimal transport problem doing the job - , the drawback is that the problem should have a unique natural reference mode.

\section{Illustration on the viscous Burger's equation in one dimension}

The viscous Burger's equation has already received some attention in the reduced modeling context. We mention [23] for the stationary case and when the solution manifolds can be well represented by a small finite dimensional linear space, withough any preconditioning.

We work in $\Omega=(-1,1)$, and solve for the time dependent viscous Burger equation with no forcing term and periodic boundary conditions (we will see later why these are important in our analysis). The extension to non periodic boundary conditions is under control and will be presented in a future paper (see also [4]).

$$
\left\{\begin{array}{l}
u_{t}+v u u_{x}-\varepsilon u_{x x}=0 \text { in }[0, T] \times \Omega \\
\left.u\right|_{t=0}=u_{0} \\
u \text { periodic }
\end{array}\right.
$$

The parameters of this problem are the triplets: $\mu=\left(u_{0}, v, \varepsilon\right)$. We want to choose a parameter domain $\mathscr{C}$ so that the problem is

- convection dominated so that the solution manifold has a large Kolmogorov nwidth

- not too stiff so as not to be bothered by stabilization issues as mentioned in the introduction, hence, we shall only consider the cases $\varepsilon \geq \varepsilon_{0}>0$ (see the recent [15] that tackles this problem).

We have chosen the following

$$
\mathscr{C}=\left\{\begin{array}{l}
\lambda \in[0.5,1.3], \\
v \in[4 ., 6 .], \\
\varepsilon \in[0.04,0.2] .
\end{array}\right.
$$

\subsection{Variational formulation and truth approximation}

For the truth approximation to the solution of problem (21), let us consider a semi implicit scheme (so as not to be bothered by a two stringent stability constraint) with time step $d t_{\text {truth }}$. Let

$$
X=H_{p e r}^{1}(\Omega)
$$

and let us denote by $\langle\cdot, \cdot\rangle$ and $\|\cdot\|$ the usual $L^{2}$ inner product and norm. For each $\mu=\left(u_{0}, v, \varepsilon\right) \in \mathscr{C}$, for the semi-discrete (in time) truth problem, we are looking for 
$u^{n+1} \in X$ (approximation of $\left.u\left(.,(n+1) d t_{\text {truth }}, \mu\right)\right)$ such that: $\forall v \in X$

$$
\left\langle u^{n+1}(\mu), v\right\rangle+d t_{\text {truth }} \varepsilon a\left(u^{n+1}(\mu), v\right)=\left\langle u^{n}(\mu), v\right\rangle-d t_{\text {truth }} v c\left(u^{n}(\mu), u^{n}(\mu), v\right)
$$

where

$$
c(w, z, v)=\int_{\Omega} w z_{x} v \quad \text { and } \quad a(w, v)=\int_{\Omega} w_{x} v_{x}
$$

This semi discretized problem is trivially well posed. In order to finalize the discretization, let us introduce an appropriate finite element discretization, the truth approximation space, $X^{\mathscr{N}}$. We pick it fine enough so that, with the chosen time step $d t_{\text {truth }}$, it is able to represent well our solution manifold. From now on, we will consider that the exact solution $u(\cdot, t ; \mu)$ and the "truth" solution $u^{\mathcal{N}}(\cdot, t, \mu)$ cannot be distinguished.

\subsection{Model order reduction - offline stage}

As mentioned earlier, the first question we need to answer is: does our solution manifold $\mathscr{M}_{\mathscr{C}}^{x}$ (in practice represented by $\mathscr{M}_{\mathscr{C}}^{x, \text { truth }}$ ) have a large Kolmogorov nwidth? And if so, can we find better behaved "calibrated" manifold solution? Figure 1 shows some snapshots $\left\{u\left(\cdot, t^{k} ; \mu\right), k \in 1 \ldots K\right\}$ taken in $\mathscr{M}_{\mathscr{C}}^{x}$ for some parameters.

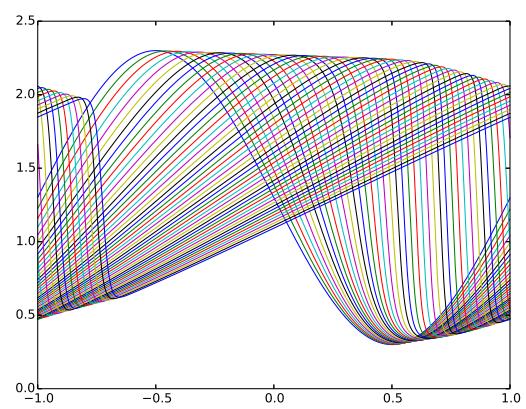

Fig. 1: Snapshots of the solution to the unsteady viscous Burger equation with $u_{0}=\lambda+\sin (x)$, $\lambda=1.3, v=4, \varepsilon=0.04$

From basic expertise on the Burger's equation, we choose the following mapping family: $\mathscr{F}=\left\{F_{t ; \mu}\right\}$, where $F_{t ; \mu}$ are defined as translation operators:

$$
\begin{aligned}
F_{t ; \mu}: \Omega & \mapsto \Omega \\
x & \mapsto x-\gamma(t ; \mu)
\end{aligned}
$$


With this choice, our family of mappings is a one parameter family, i.e:

$$
\mathscr{F}=\{F(\gamma), \gamma \in \mathbb{R}\} .
$$

Unlike in the pure translation problem of the introduction (4), our parameter $\gamma$ is not constant (it is a function of $\mu$ and time) and has no analytical expression. One possible calibration (and the most natural one) is presented on Figure 2. where we pick $\gamma$ manually so that all steepest points coincide. Our calibrated solution manifold is then

$$
\mathscr{M}_{\mathscr{F}, \mathscr{C}}^{x}=\{u(\cdot-\gamma(t ; \mu), t ; \mu), t \text { in }[0, T], \mu \in \mathscr{C}\},
$$

that is represented in Figure 2 where we understand that the Kolmogorov $n$-width of $\mathscr{M}_{\mathscr{F}, \mathscr{C}}^{x}$ is smaller than the original one represented in Figure 1.

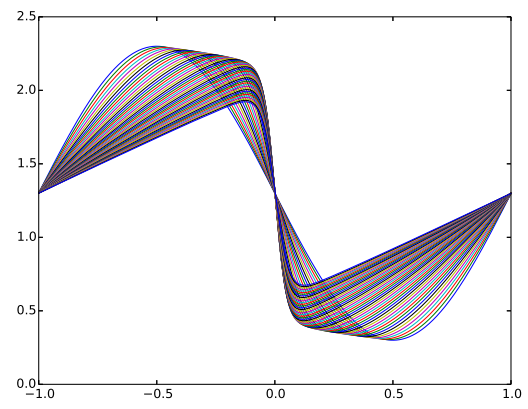

Fig. 2: Calibrated set of the above snapshots for $u_{0}=\lambda+\sin (x), v=4, \varepsilon=0.04$

This is confirmed in Figure 3 which presents the decay of the POD eigenvalues in logarithmic scale for $\mathscr{M}_{\mathscr{C}}^{x}$ and $\mathscr{M}_{\mathscr{F}, \mathscr{C}}^{x}$. As we could have expected, to achieve a fixed accuracy, the number of POD modes needed to represent the calibrated manifold is much smaller than the number of modes needed for the original solution set. To confirm this, we present on Figure 4 the 3rd and 6th POD modes of the calibrated and non calibrated simulations. As we can see, in the calibrated case, with just 3 modes, our $L^{2}$-projection focuses on reproducing the shock, whereas in the non calibrated case, the modes desperatly try to represent shocks centered anywhere in $\Omega$. We mention again the fact that even in the calibrated case, our algorithm could be improved using $L^{1}$-minimization. We present on Figure 5 the projection of one of the snapshot on the first three POD modes. With 10 POD modes in the uncalibrated case, the projection shown on Figure 5 exhibit the oscillatory behaviour as described in [1].

At this stage, we suppose that we have found a "calibrated" solution manifold, with nice Kolmogorov n-width decay. That is, we have calibrated an original dataset, and obtained a reduced orthonormal basis: 


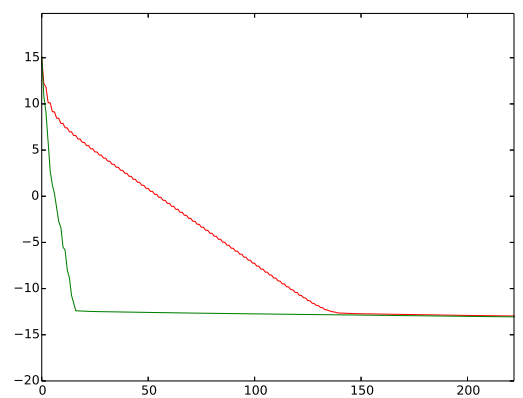

Fig. 3: Eigenvalues of the POD decomposition of the original set of snapshots ( in red) and of the calibrated set of snapshots (in green)
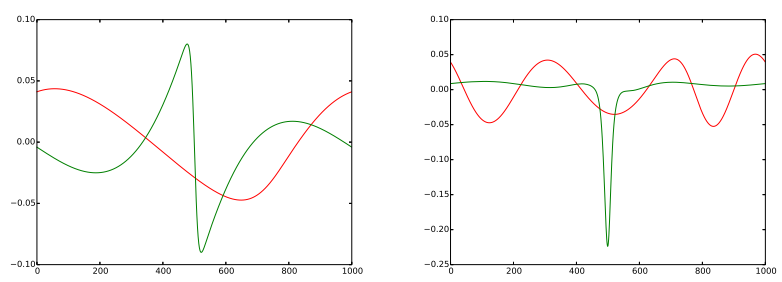

Fig. 4: 3rd (left) and 6th (right) POD modes for the calibrated (green) and original (red) simulations
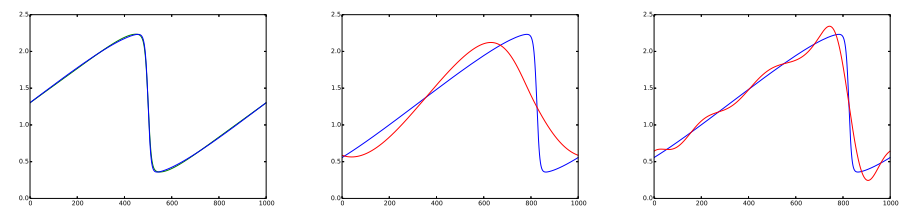

Fig. 5: Projection of a snapshot (blue) on:

- left: 3 POD modes in the calibrated case

- center: 3 POD modes in the non calibrated case

- right: 10 POD modes in the non calibrated case

$$
\operatorname{span}\left\{\phi_{i}, i=1 \ldots M\right\} \subset X
$$

that approximates well the calibrated solution manifold $\mathscr{M}_{\mathscr{F}, \mathscr{C}}^{x}$.

We now need to explicit the algorithm presented in the previous section. The biggest question is how do we pick the $F \in \mathscr{F}$ at each time step? 


\subsection{Model order reduction - online stage}

As was introduced in the previous section (see (12)), for the time semi-discretization of the RBM approach, we use a forward Euler discretization with a time step $d t$ that may be different from $d t_{\text {truth }}$, at each time step we are looking for the solution to the following elliptic problem ${ }^{2}$ :

$$
u^{n+1}=u^{n}-d t v u^{n} u_{x}^{n}+d t \varepsilon u_{x x}^{n}
$$

with periodic boundary conditions over $(-1,1)$, which leads to the following variational formulation that will be used to provide the Galerkin formulation of the RBM: knowing $u^{n}$, compute $u^{n+1} \in X$ such that

$$
\forall v \in X,\left\langle u^{n+1}(\mu), v\right\rangle=\left\langle u^{n}(\mu), v\right\rangle-d t v c\left(u^{n}(\mu), u^{n}(\mu), v\right)-d t \varepsilon a\left(u^{n}(\mu), v\right) \text {. }
$$

One could fear that a problem with this discretization is the stringent CFL condition on the time-step. Our reduced basis formulation will allow for very fast computation, which will mitigate this issue on which we shall dwell upon later. As said already, we could also consider an implicit Euler scheme. We refer to [23] (stationary) and [18] (non stationary), for the development of reduced order model in that case.

The full RBM discretization starts from the knowledge of the (supposedly accurate) approximation of $u^{n}$ as an expansion

$$
u^{n}:=\sum_{i=1}^{M} \alpha_{i}^{n} \phi_{i} \circ F_{n},
$$

where the $\left\{\phi_{i}\right\}_{i}$ are the reduced basis elements of the good approximation of the calibrated solution manifold that have been introduced in (29) as a result of the offline process. $F_{n}$ is here $F\left(\gamma^{n}\right)$ where $\gamma^{n}$ is the current translation value. In order to deduce the next approximation,

$$
u^{n+1}:=\sum_{i=1}^{M} \alpha_{i}^{n+1} \phi_{i} \circ F_{n+1}, \text { where } F_{n+1}=F\left(\gamma_{n+1}\right)
$$

as described in the previous section, we iterate between the search for the reduced coordinates $\left(\alpha_{i}^{n+1}\right)_{i}$ and for the mapping $F_{n+1}$ i.e. for the translation parameter $\gamma^{n+1}$, we initialize these entities as follows:

$$
\begin{aligned}
& \alpha_{i}^{n+1,0}=\alpha_{i}^{n} \\
& \gamma^{n+1,0}=\gamma^{n}+\left(\gamma^{n}-\gamma^{n-1}\right) .
\end{aligned}
$$

\footnotetext{
${ }^{2}$ Indeed there is no reason why using the same discretization in time for the truth solution and for the reduced basis scheme
} 
In the first part of the iterative step indexed by $q$, assuming we know $\left(\left(\alpha_{i}^{n+1, q}\right)_{i}, \gamma^{n+1, q}\right)$ we fit the $\alpha_{i}$ for a fixed translation parameter $\gamma$, i.e.we are looking for $\left(\alpha_{i}^{n+1, q+1}\right)_{i}$ that satisfy

$$
\left\{\alpha_{i}^{n+1, q+1}\right\}=\underset{\left(\alpha_{i}\right)_{i} \in \mathbb{R}^{\mathbb{N}}}{\operatorname{argmin}}\left\|\sum_{i} \alpha_{i} \phi_{i} \circ F\left(\gamma^{n+1, q}\right)-u^{n}-d t v u^{n} u_{x}^{n}+d t \varepsilon u_{x x}^{n}\right\|_{2}^{2}
$$

The nice feature with the chosen norm is that we pick our reduced coordinates such that our residual is orthogonal to the translated reduced space, the space spanned by the $\left\{\phi_{i} \circ F\left(\gamma^{n+1, q}\right)\right\}_{i}$. Using $u^{n}$ 's expansion on its reduced basis, the coefficients $\left\{\alpha_{i}^{n+1, q+1}\right\}_{i}$ are given by the first-order optimality condition:

$$
\begin{aligned}
\alpha_{i}^{n+1, q+1} & =\sum_{j} \alpha_{j}^{n}\left\langle\phi_{j} \circ F\left(\gamma^{n}\right), \phi_{i} \circ F\left(\gamma^{n+1, q}\right)\right\rangle \\
& -d t v \sum_{j} \sum_{p} \alpha_{j}^{n} \alpha_{p}^{n}\left\langle\phi_{j} \circ F\left(\gamma^{n}\right)\left(\phi_{p} \circ F\left(\gamma^{n}\right)\right)_{x}, \phi_{i} \circ F\left(\gamma^{n+1, q}\right)\right\rangle \\
& -d t \varepsilon \sum_{j} \alpha_{j}^{n}\left\langle\left(\phi_{j} \circ F\left(\gamma^{n}\right)\right)_{x},\left(\phi_{i} \circ F\left(\gamma^{n+1, q}\right)\right)_{x}\right\rangle .
\end{aligned}
$$

In order to evaluate this expression, we need to compute the following integrals:

$$
\begin{cases}\forall i, j, & \int_{\Omega} \phi_{j} \circ F\left(\gamma^{n}\right)(x) \phi_{i} \circ F\left(\gamma^{n+1, q}\right)(x) \\ \forall i, j, p, & \int_{\Omega} \phi_{j} \circ F\left(\gamma^{n}\right)(x)\left(\phi_{p} \circ F\left(\gamma^{n}\right)\right)_{x}(x) \phi_{i} \circ F\left(\gamma^{n+1, q}\right)(x) \\ \forall i, j, & \int_{\Omega} \phi_{j} \circ F\left(\gamma^{n}\right)_{x}(x) \phi_{i} \circ F\left(\gamma^{n+1, q}\right)_{x}(x)\end{cases}
$$

We will see in the next subsection how to achieve efficient offline/online decomposition for these quantities.

Once this is done, we fit the $\gamma$. Let us define first the residual function $r(\gamma)$ :

$$
r(\gamma)=\left\|\sum_{i} \alpha_{i}^{n+1, q+1} \phi_{i} \circ F_{\gamma}-u^{n}-d t v u^{n} u_{x}^{n}+d t \varepsilon u_{x x}^{n}\right\|_{2}^{2},
$$

then we choose $\gamma^{n+1, q+1}$ as the "best", i.e residual minimizing, translation parameter. It is given by:

$$
\gamma^{n+1, q+1}=\underset{\gamma}{\operatorname{argmin}} r(\gamma)
$$

Next we develop $r(\gamma)$ :

$$
\begin{array}{r}
r(\gamma)=\left\|\sum_{i} \alpha_{i}^{n+1, q+1} \phi_{i} \circ F(\gamma)\right\|_{2}^{2}+\left\|u^{n}-d t v u^{n} u_{x}^{n}+d t \varepsilon u_{x x}^{n}\right\|_{2}^{2} \\
-2\left\langle\sum_{i} \alpha_{i}^{n+1, q+1} \phi_{i} \circ F(\gamma), u^{n}-d t v u^{n} u_{x}^{n}+d t \varepsilon u_{x x}^{n}\right\rangle .
\end{array}
$$

The second term is independent of $\gamma$. The first one, using periodicity, happens also to be independent of $\gamma$. We can thus replace the minimization of $r$ by the minimisation of the following quantity $\tilde{r}$ : 


$$
\tilde{r}(\gamma)=-\left\langle\sum_{i} \alpha_{i}^{n+1, q+1} \phi_{i} \circ F(\gamma), u^{n}-d t v u^{n} u_{x}^{n}+d t \varepsilon u_{x x}^{n}\right\rangle .
$$

Here again, we we need to evaluate the quantities

$$
\begin{cases}\forall i, j, & \int_{\Omega} \phi_{j} \circ F\left(\gamma^{n}\right)(x) \phi_{i} \circ F(\gamma)(x) \\ \forall i, j, p, & \int_{\Omega} \phi_{j} \circ F\left(\gamma^{n}\right)(x)\left(\phi_{p} \circ F\left(\gamma^{n}\right)\right)_{x}(x) \phi_{i} \circ F(\gamma)(x) \\ \forall i, j, & \int_{\Omega} \phi_{j} \circ F\left(\gamma^{n}\right)_{x}(x) \phi_{i} \circ F(\gamma)_{x}(x)\end{cases}
$$

for various values of $\gamma$ in order to derive the value of $\gamma$ that minimizes $r$ (or $\tilde{r}$ ).

\subsection{Offline/Online decomposition of the expressions depending on}

$\gamma$

In both the search for $\gamma\left(\right.$ see (41)) and $\left(\alpha_{i}\right)_{i}$ (see (37)), we need to compute scalar products of the form:

$$
\left\langle\psi_{i} \circ F\left(\gamma^{n}\right), \psi_{j} \circ F(\gamma)\right\rangle
$$

where $\psi$ can be one of the POD basis or one of its $x$-derivatives. $\gamma^{n}$ and $\gamma$ can take any value in $\Omega$. Our key ingredient here is that, due to translation invariance (because we are in a periodic settings), we can replace the previous terms by

$$
\left\langle\psi_{i} \circ F\left(\gamma^{n}-\gamma\right), \psi_{j}\right\rangle=\left\langle\psi_{i} \circ F(\Delta \gamma), \psi_{j}\right\rangle .
$$

We have plotted on Figure 6 these quantities (after rescaling) as a function of $\Delta \gamma$ for some pairs of chosen $\psi$ 's and we notice that, as can be expected because we are essentially using a primitive function of the integrant, these are regular functions of $\Delta \gamma$.

For a sufficiently small time step, we expect $\Delta \gamma$ to be of order $d t * c$ where $c$ is some local characteristic velocity. We have chosen the following method:

- precompute the scalar products for a predefined set of values of $\Delta \gamma$

- using some regularity hypothesis, use spline interpolation to get approximated values for all $\gamma$ in $\left[-d t * c_{\max }, d t * c_{\max }\right]$, where $c_{\max }$ is the maximum expected shock speed during the simulation.

Remark 2. For the optimization of $\tilde{r}$ we have also tested to linearize our problem around $\gamma^{n}$ which leads to a doable method but does not work better that the above. 


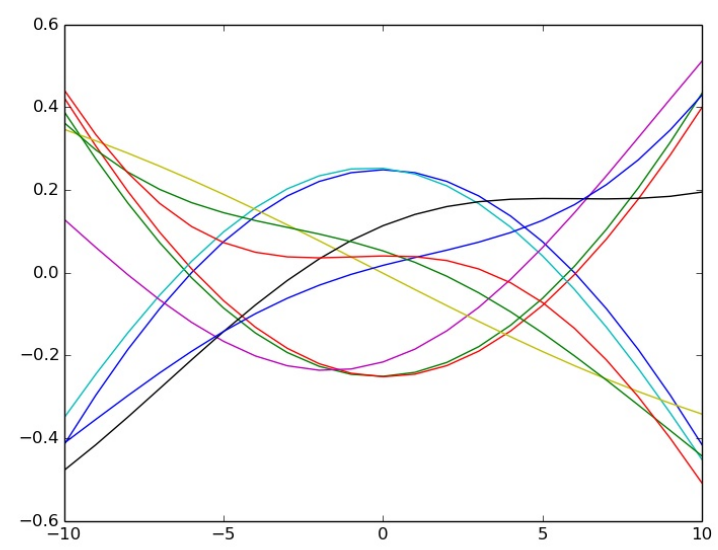

Fig. 6: A few values of the quantities (43) as a function of $\Delta \gamma$. The $x$ axis is scaled to multiples of $c * \Delta t$.

\section{Numerical results}

\subsection{About the CFL Condition}

We represent in Figure 7 the value of the CFL condition of our reduced scheme using the space calibrated $\mathscr{M}_{\mathscr{F} \mathscr{C}}^{x}$ as a function of the dimension $M$ of the discrete space expressed in Equation (32). Of course, the bigger the reduced basis, the smaller the time step required for stability. We remark that there is a plateau for large values of $M$ that is above the CFL-condition for the truth solver. More importantly, for $M=5$, we can use a discrete time step 3,000 times bigger than the one of the fine (finite element) scheme (that was $d t_{\text {truth }} \leq 10^{-6}$ ).

\subsection{Convergence Illustration}

On the next Figure 8, we have plotted the $L^{2}$-error of the solution of (15) in case of problem (21) as a function of time for different values of the reduced basis for $d t=2.510^{-4}$. The different colors represent various values of $M$ used in (32) (Note that on the same figure, the plots close the $x$ axis represent the projection errors of the solution onto the set $\mathscr{M}_{\mathscr{F}, \mathscr{C}}^{x}$ with the exact value of the translation $\gamma$ ). We see that our numerical scheme is convergent, as a function of $M$. The final accuracy is somehow difficult to grasp since it is a function of $\Delta t$ and the number of degrees of freedom used in the spatial direction (here $M$ ) as for any discretization of an 


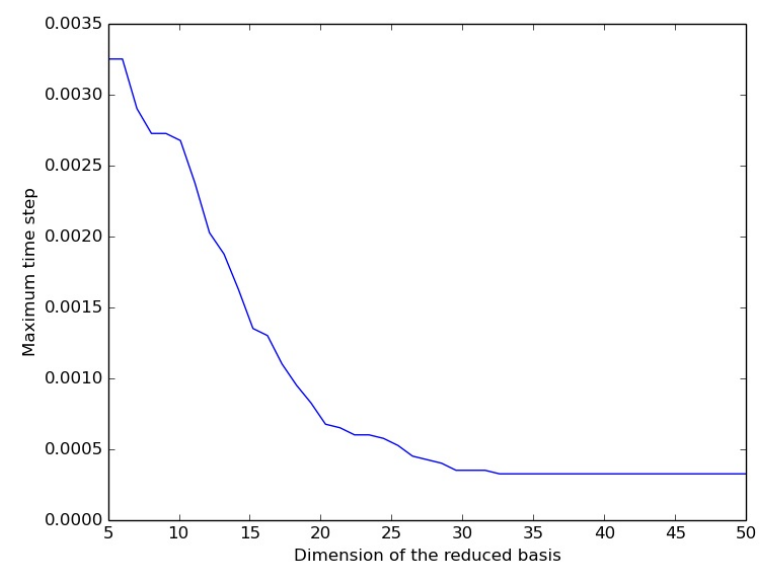

Fig. 7: A few values of the quantities (43) as a function of $\Delta \gamma$. The $x$ axis is scaled to multiples of $c * \Delta t$.

evolution problem. It is however also a function of the way the value of $\gamma$ is found as each time step as a solution of the full minimization problem (15). As one can expect, the maximum of the error is decreasing as $M$ increases.

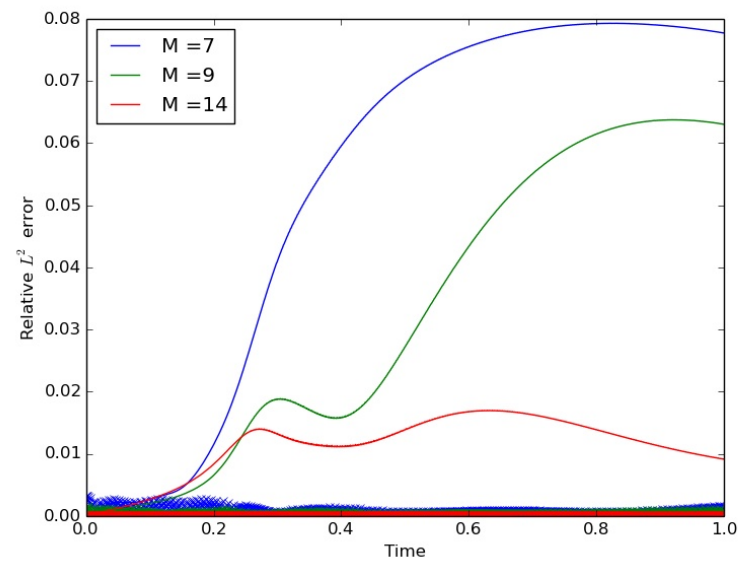

Fig. 8: Relative $L^{2}$-error of the solution as a function of time for different values of the reduced basis. 


\section{Conclusion}

This paper is the first of a series that explain how to correct the impossibility of the standard reduced basis method (or actually most model reduction methods) to approximate well convection dominated phenomenon. The additional ingredient is to propose a change of variable, that should also be represented by few coefficients, that are updated thanks to the numerical scheme that is used classically for the discretization of the convection dominated problem. This simple approach can be implemented in an online/offline paradigm that allows online to contribute with a complexity that, at each time step, is a function of the number of reduced basis that are used for the approximation. This paper that allows to set the scene of this new approach deals with a problem with periodic boundary conditions to focus on the main feature of the approach.

The reduced basis here is composed of snapshots of the solution. Note that we could also use the gradient of these snapshots in order to diminish the effect of the mismatched of the correct value of $\gamma$ in the iterative process used to solve (15). In the toy problem used here this does not improve the accuracy but in multidimensional situations it may be useful, more tests on this are under investigations.

Acknowledgement This paper is dedicated to Professor Olivier Pironneau on the occasion of his 70th birthday. The French government is greatly acknowledged for its funding of the FUI MECASIF project.

\section{References}

1. Abgrall, R. and Amsallem, D., "Robust Model Reduction by L-norm Minimization and Approximation via Dictionaries: Application to Linear and Nonlinear Hyperbolic Problems", preprint, 2015.

2. Beyn, W.-J. and Thümmler, V., "Freezing solutions of equivariant evolution equations", SIAM Journal on Applied Dynamical Systems, 3, 2, pp 85-116, (2004

3. Binev, P. and Cohen, A. and Dahmen, W. and DeVore, R. and Petrova, G. and Wojtaszczyk, P., "Convergence rates for greedy algorithms in reduced basis methods", SIAM Journal on Mathematical Analysis, 43, 3, pp 1457-1472, 2011.

4. Cagniart, N. PhD thesis, in preparation at University Pierre et Marie Curie (2017).

5. Carlberg, K. "Adaptive h-refinement for reduced-order models", International Journal for Numerical Methods in Engineering, 102, 5, pp 1192-1210, (2015).

6. Carlberg, K. and Bou-Mosleh, C. and Farhat,C., "Efficient Nonlinear Model Reduction via a Least-Squares Petrov-Galerkin Projection and Compressive Tensor Approximations", International Journal for Numerical Methods in Engineering, Vol. 86, pp. 155-181 (2011)

7. Carlberg, K. and Farhat, C. and Cortial, J. and Amsallem, D. , "The GNAT Method for Nonlinear Model Reduction: Effective Implementation and Application to Computational Fluid Dynamics and Turbulent Flows", Journal of Computational Physics, Vol. 242, pp. 623-647 (2013)

8. Cohen, A. and DeVore, R., "Kolmogorov widths under holomorphic mappings", IMA Journal of Numerical Analysis, (2015). 
9. Dahmen, W. and Plesken, C. and Welper, G., "Double greedy algorithms: reduced basis methods for transport dominated problems", ESAIM: Mathematical Modelling and Numerical Analysis, 48, 3, pp 623-663 (2014).

10. , DeVore, R. and Petrova, G. and Wojtaszczyk, P., "Greedy algorithms for reduced bases in Banach spaces", Constructive Approximation, 37, 3, pp 455-466, (2013).

11. Gerbeau, J.-F. and Lombardi, D., "Approximated Lax pairs for the reduced order integration of nonlinear evolution equations", Journal of Computational Physics, 265, pp. 246-269, (2014).

12. Hesthaven, J.S. and Rozza, G. and Stamm, B. , "Certified Reduced Basis Methods for Parametrized Partial Differential Equations". SpringerBriefs in Mathematics. Springer, 2016.

13. Iollo, A. and Lombardi, D., "Advection modes by optimal mass transfer", Physical Review E, 89, 2, pp 022923 (2014).

14. Løvgren, A. E., and Maday, Y., and Rønquist, E. M. "A reduced basis element method for the steady Stokes problem”. ESAIM: Mathematical Modelling and Numerical Analysis, 40(3), pp 529-552. 2006.

15. Maday, Y. and Manzoni ,A. and Quarteroni, A., "An online intrinsic stabilization strategy for the reduced basis approximation of parametrized advection-dominated problems", Comptes Rendus Mathematique, (in press).

16. Maday, Y. and Patera, A.-T. and Turinici, G., "A priori convergence theory for reduced-basis approximations of single-parameter elliptic partial differential equations", Journal of Scientific Computing, 17, 1-4, pp 437-446, 2002.

17. Melenk, J. -M, . "On n-widths for elliptic problems, Journal of mathematical analysis and applications", 247, 1, pp 272-289, 2000

18. Ngoc-Cuong, N. "Reduced basis approximation and a posteriori error estimation for the timedependent viscous Burgers's equation”, Calcolo , 46, 3, pp 157-185, (2009)

19. Ohlberger, M. and Rave, S., "Nonlinear reduced basis approximation of parameterized evolution equations via the method of freezing" Comptes Rendus Mathématique, 351, 23, pp 901-906, (2013).

20. Quarteroni, A. and Manzoni, A. and Negri, F. , "Reduced Basis Methods for Partial Differential Equations. An Introduction”, volume 92 of Unitext Series, Springer, 2016.

21. Sirovich, L., "Turbulence and the dynamics of coherent structures. Part I: Coherent structures", Quarterly of applied mathematics, 45, 3, pp 561-571, (1987).

22. Taddei, T and Perotto, S and Quarteroni, A : "Reduced basis techniques for nonlinear conservation laws", , ESAIM: Mathematical Modelling and Numerical Analysis, 49, 3, pp. 787-814, (2015)

23. Veroy, K. and Prud'homme, C. and Patera, A.-T., "Reduced-basis approximation of the viscous Burgers equation: rigorous a posteriori error bounds", Comptes Rendus Mathematique, 337, 9, pp 619-624 (2003). 